\title{
Rôle des accélérateurs de particules dans la lutte contre le SARS-CoV-2
}

Sam Bayat ${ }^{(1)}$ (sbayat@chu-grenoble.fr) et Nicolas Delerue ${ }^{(2)}$ (delerue@lal.in2p3.fr)

(1) STROBE (Rayonnement synchrotron pour la recherche médicale),

Université Grenoble Alpes, INSERM UA 07

CHU Grenoble Alpes, LPSC, 53 Avenue des Martyrs, 38026 Grenoble Cedex

(2) Laboratoire de physique des 2 infinis - Irène Joliot-Curie (IJCLab),

Université Paris-Sud, Bât. 100, 91405 Orsay Cedex

À travers le monde, les équipes sanitaires ont été en première ligne pour lutter contre la propagation du virus SARS-CoV-2, responsable de la pandémie de Covid-19. Au-delà du traitement de l'urgence médicale, des recherches sont nécessaires pour mieux comprendre comment inhiber les mécanismes d'action du virus.

\section{On peut, entre autres, imager} par diffraction la structure de ses protéines grâce au rayonnement $X$ synchrotron ou à des flux de neutrons, ou étudier les lésions qui se forment dans les poumons.

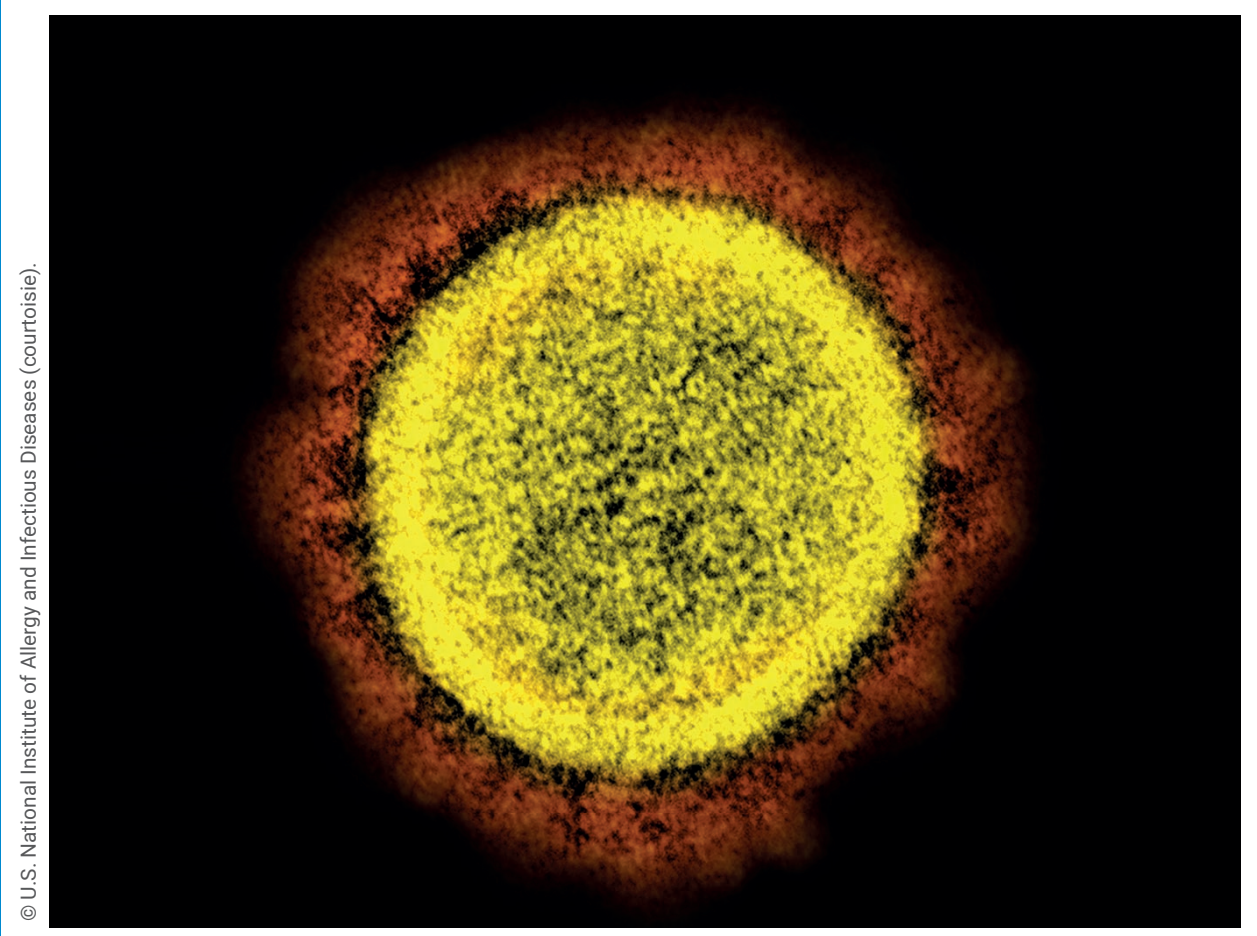

1. Micrographie d'un virion de SARS-CoV-2 isolé sur un patient, obtenue par microscopie électronique en transmission. La couronne (colorisée en rouge) est formée de protéines qui permettent au virus de se frayer un chemin dans les cellules hôtes. Image réalisée et colorisée dans le laboratoire NIAID Integrated Research Facility de Fort Derrick (Maryland).

En décembre 2019, une épidémie de pneumonies d'étiologie inconnue a émergé dans la ville de Wuhan (capitale de la province de Hubei, Chine) et, depuis, s'est rapidement propagée à travers le monde. Dès janvier 2020, le virus à l'origine de ces pneumonies est identifié, puis la séquence complète de son génome est établie. Les chercheurs des centres de rayonnement synchrotron et des sources de neutrons ont alors décidé de mettre à profit les faisceaux intenses et pénétrants dont ils disposent pour contribuer à la lutte contre la maladie.

\section{Visualiser les protéines clés}

Pour mieux lutter contre un virus, il est important de bien le connaitre. Alors que l'épidémie de Covid-19 provoquée par le deuxième coronavirus du syndrome respiratoire aigu sévère (SARS-CoV-2) prenait de l'ampleur, des chercheurs à travers le monde se sont mobilisés afin d'imager la structure en 3D de ce virus et surtout des protéines qui lui permettent de pénétrer dans les cellules qu'il infecte et de se multiplier. 
La plupart des virus ont une taille de l'ordre de quelques dizaines à une centaine de nanomètres. Avec un diamètre de l'ordre de 125 nanomètres, le SARS-CoV-2 est assez grand et peut être observé par un microscope électronique en transmission (fig. 1). Sur ces images, le virus apparait entouré d'une couronne (en réalité une coquille sphérique), et c'est d'ailleurs ce qui lui a valu son nom (coronavirus signifie "virus à couronne "). Les protubérances sur cette couronne (fig. 2) sont des protéines S, qui permettent au virus de pénétrer dans les cellules hôtes à l'intérieur desquelles il va se répliquer.

Les protéines S (pour "Spike", pointe en anglais) jouent un rôle majeur dans la capacité des virions $^{(a)}$ du SARS-CoV-2 à infecter une cellule hôte. En effet, ce sont ces protéines $\mathrm{S}$ qui vont agir comme la "clef " qui permet au virion de pénétrer dans une cellule. Dans le cas du SARSCoV-2, il a été démontré que les protéines $\mathrm{S}$ ont une affinité forte pour des enzymes, appelées $\mathrm{ACE} 2^{(\mathrm{b})}$, se trouvant à la surface de certaines cellules du système respiratoire. Les récepteurs ACE2 sont des points d'entrée du SARS-CoV-2 dans ces cellules.

Les protéines étant trop petites pour être imagées par microscopie électronique, il a fallu recourir à des accélérateurs de particules utilisés comme sources de rayonnement synchrotron. Dans ces sources, les électrons relativistes qui tournent dans un anneau de stockage émettent un rayonnement électromagnétique intense, dans un spectre allant de l'infrarouge lointain aux rayons $\mathrm{X}$ durs. La forte intensité de ce rayonnement permet de déterminer précisément par diffraction de rayons $\mathrm{X}$ la structure de nombreux cristaux (encadré 1).

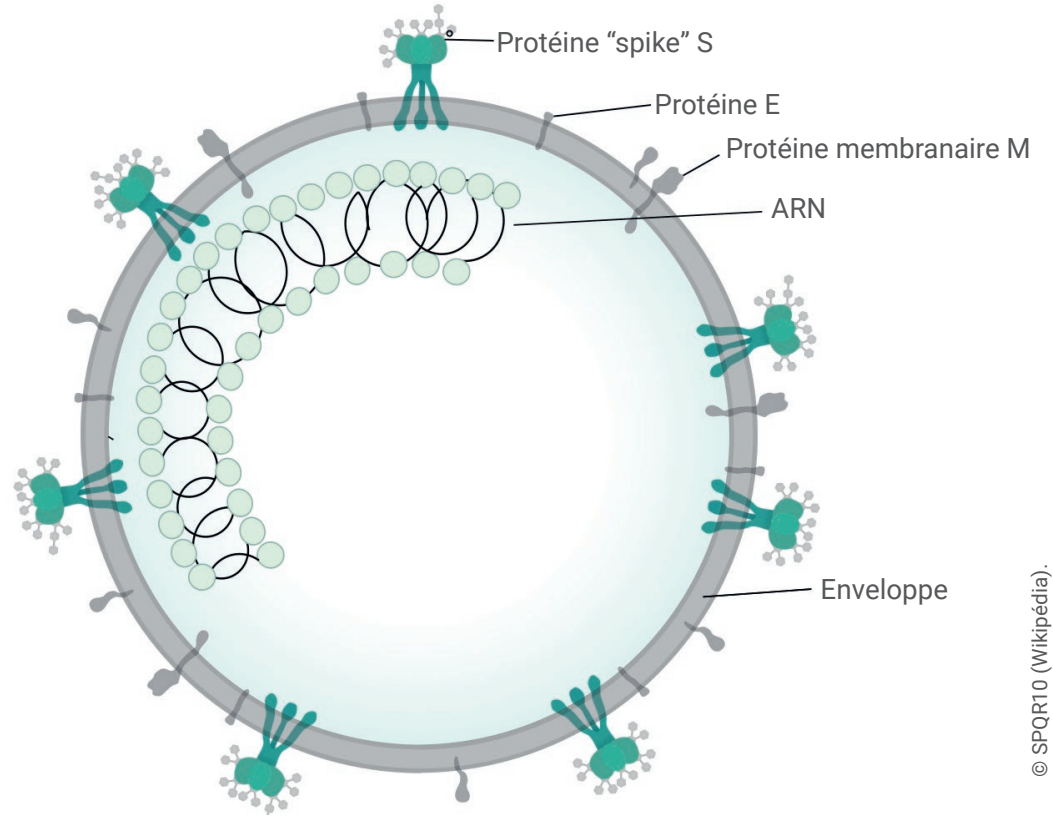

2. Schéma de la structure du virion SARS-CoV-2. Les protéines $S, E$ et $M$ sont insérées dans l'enveloppe virale lipidique. L'ARN du virus se trouve à l'intérieur du virion.

\section{La cristallographie sur sources de rayonnement synchrotron}

Encadré 1

Afin de déterminer la structure d'un cristal, on le soumet à un flux de rayons $X$ et on regarde le motif produit de l'autre côté du cristal. En effet, les rayons $X$ sont diffractés par la structure cristalline et ils interférent entre eux. Le motif observé, appelé « spectre de diffraction ", dépend de la maille cristalline et de la longueur d'onde des rayons X utilisés. En faisant tourner l'échantillon par rapport à la source de rayons $X$, l'angle avec lequel ces derniers arrivent sur la maille cristalline change et le motif change aussi. Grâce à plusieurs mesures effectuées à des angles différents, il est possible de déterminer les paramètres de la maille cristalline, ainsi que les positions et (dans une certaine mesure) la nature des atomes. En fait, le rayonnement $X$ interagit avec le nuage électronique du matériau étudié, et ce qui est réellement mesuré à partir de l'intensité du spectre de diffraction est donc la distribution des électrons dans le cristal.

Cette technique, très bien maitrisée, est utilisée de manière routinière sur tous les diffractomètres installés sur les lignes de rayonnement synchrotron pour étudier des molécules complexes telles que des protéines (à condition de pouvoir les cristalliser) [1].
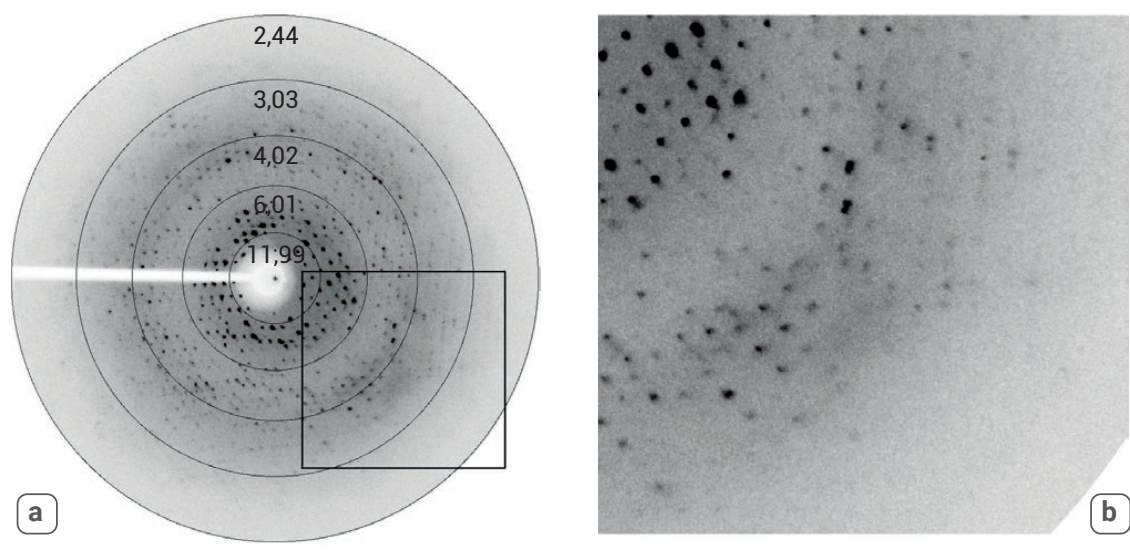

E1. (a) Exemple de motif de diffraction de rayons $X$ obtenu sur un cristal d'une protéine de SARS-CoV, observé lors de l'épidémie de 2004. (b) Image élargie de la zone encadrée sur la figure (a). Source : Yanhui Xu et al., Acta Cryst D 60 (2004) 2377-2379. 


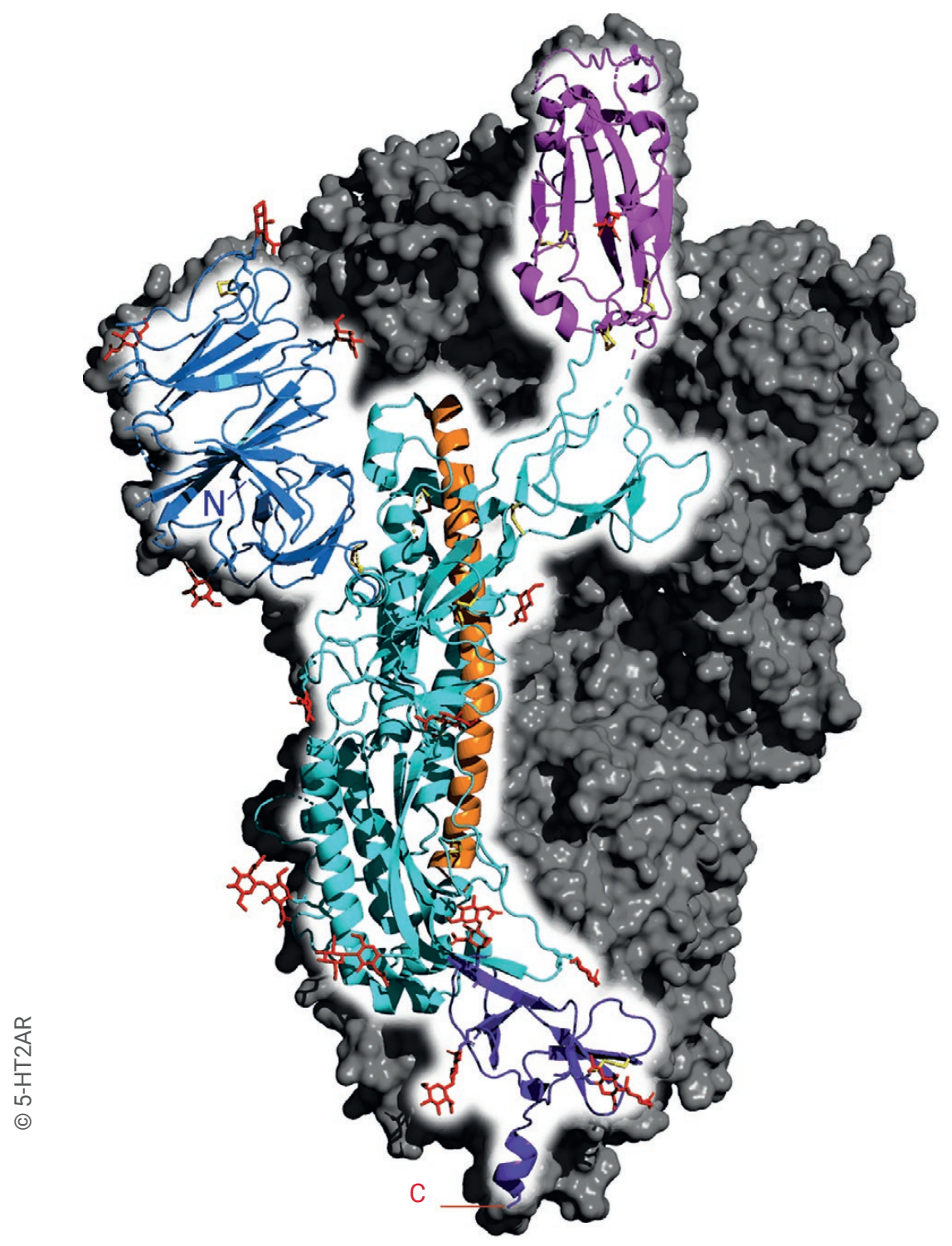

3. Représentation de la protéine de pointe du SARS-CoV-2. La protéine $S$ entière est formée de trois parties identiques dont une seule est mise en évidence ici, les deux autres parties étant représentées en gris. Le domaine de la protéine permettant de se lier au récepteur ACE2 est représenté en magenta en haut de l'image.

\section{Actions spécifiques du synchrotron SOLEIL}

Encadré 2

\section{dans la lutte contre la pandémie Covid-19}

Le synchrotron SOLEIL, situé sur le plateau de Saclay, possède plusieurs lignes de lumière participant aux recherches sur la Covid-19 (lignes de bio-cristallographie, Bio-SAXS, imageries $\mathrm{X}, \mathrm{X}$-mous, ultraviolet lointain et infrarouge). Certaines avaient déjà contribué aux efforts contre les virus chikungunya, dengue et zika, en collaboration avec des virologues de l'Institut Pasteur. Un système d'accès rapide a été mis en place, afin de fluidifier les demandes de temps d'expérimentation dans le cadre de la lutte contre la pandémie.

Un consortium regroupant le synchrotron SOLEIL, I'INRAe et SANOFI a obtenu un financement de l'ANR sur cette thématique. Il est possible de suivre l'évolution de ce projet sur la page www.synchrotron-soleil.fr/en/beamlines/proxima-1/acces-ge-covid-19 .
$>>>$

Dès la compréhension de l'importance de la protéine $\mathrm{S}$ et de son mécanisme de liaison avec les récepteurs ACE2, cette protéine et ce mécanisme ont été étudiés grâce au rayonnement synchrotron (fig. 3). Il était en particulier important de comprendre à quel site de l'enzyme ACE2 se lie la protéine $\mathrm{S}$. Une première étude en ce sens a été réalisée dès le mois de janvier 2020 et publiée dans Nature au mois de mars [2]. Lors de cette étude, le gène codant la protéine $\mathrm{S}$ a été introduit par un autre virus dans des cellules en cultures, permettant ainsi la production d'une quantité suffisante de protéine $\mathrm{S}$ pour permettre son imagerie.

Plusieurs études ont été réalisées sur accélérateur à la même période, afin de comprendre la structure d'autres protéines jouant cette fois-ci un rôle important dans la réplication du virus SARS-CoV-2. En particulier, dans une étude publiée dans Science en mars 2020, la structure de la protéase $^{(\mathrm{c})}$ majeure $\mathrm{M}^{\text {pro }}$ du SARS-CoV-2, qui joue un rôle clé dans la multiplication du virus à l'intérieur d'une cellule, a été déterminée par diffraction de rayons $\mathrm{X}$ sur la source synchrotron BESSY II à Berlin [3].

Une fois la structure d'une protéine comprise, elle peut être diffusée dans la communauté scientifique par le biais d'une base de données spécialisée [4]. À partir de cette base de données, il est possible d'utiliser des outils de biologie moléculaire afin de reproduire une protéine dont on connait la structure même sans avoir accès au virus lui-même, ce qui est un atout non négligeable quand il s'agit d'un virus contagieux. Des chercheurs ont utilisé la structure ainsi publiée de la protéase $M^{\text {pro }}$ citée ci-dessus pour la reproduire en laboratoire. Une fois cette enzyme reproduite, il a été possible de la confronter avec une bibliothèque de plus de 10000 fragments de molécules utilisées en pharmacologie, afin de voir si certains d'entre eux étaient capables d'empêcher son action et donc d'arrêter la multiplication du SARSCoV-2. Cela a débouché sur l'identification de six candidats qui pourraient empêcher cette réplication, dont un avec une action antivirale apparente [5].

Plus largement à travers le monde, de nombreuses sources de lumière synchrotron dont les deux situées en France, SOLEIL à Saclay (encadré 2) et l'ESRF à Grenoble, ont lancé des appels à projets spécifiques suite à la crise sanitaire pour permettre des études liées au SARS-CoV-2 [6]. Une 
visioconférence a eu lieu courant avril 2020, afin de coordonner au niveau mondial les actions contre la Covid-19.

À SOLEIL, grâce à un soutien de l'ANR (Agence nationale de la recherche), des équipes ont étudié un procédé innovant permettant de cloner et de cristalliser les protéines du virus de manière systématique et beaucoup plus rapide que par les méthodes habituelles. Pour cela, des cellules biologiques sont mises dans des conditions où certaines d'entre elles vont produire naturellement des cristaux de protéines. Plusieurs lignes de lumière sont ensuite mises à contribution. L'apparition de ces cristaux dans certains échantillons est d'abord repérée sur une première ligne de lumière, avant qu'ils ne soient apportés sur une ligne dédiée à la cristallographie où ils sont caractérisés afin de déterminer leur structure.

Il est possible de remplacer le flux de rayons $\mathrm{X}$ par un flux de neutrons. Tout comme pour celles effectuées avec des rayons $\mathrm{X}$, le but des études avec des neutrons est de trouver des molécules pouvant bloquer la réplication du SARS$\mathrm{CoV}-2$. Or les neutrons sont insensibles au nuage électronique et permettent de mesurer la distribution des noyaux atomiques dans le cristal. Ils ont l'avantage sur les photons de diffuser sur l'hydrogène atomique et sur les protons. Ils offrent ainsi une meilleure visibilité des liaisons hydrogène, qui sont des éléments importants de l'action bloquante d'une molécule. Parce qu'elles ne mesurent pas la même chose, les études cristallographiques avec des rayons $\mathrm{X}$ et des neutrons sont donc complémentaires pour comprendre la structure d'une protéine. Mais les flux de neutrons sont beaucoup plus faibles que ceux de rayons X. Pour obtenir des images de bonne qualité, les mesures par diffraction neutronique requièrent donc des cristaux plus gros et qui prennent plus de temps à être préparés que ceux utilisés pour les rayons X.

Après avoir été mises à l'arrêt pendant les premières semaines de la pandémie, plusieurs sources de neutrons (basées sur accélérateur ou sur réacteur nucléaire), en particulier le réacteur à haut flux de l'Institut Laue-Langevin à Grenoble, ont redémarré pour des études liées au SARSCoV-2 [7].
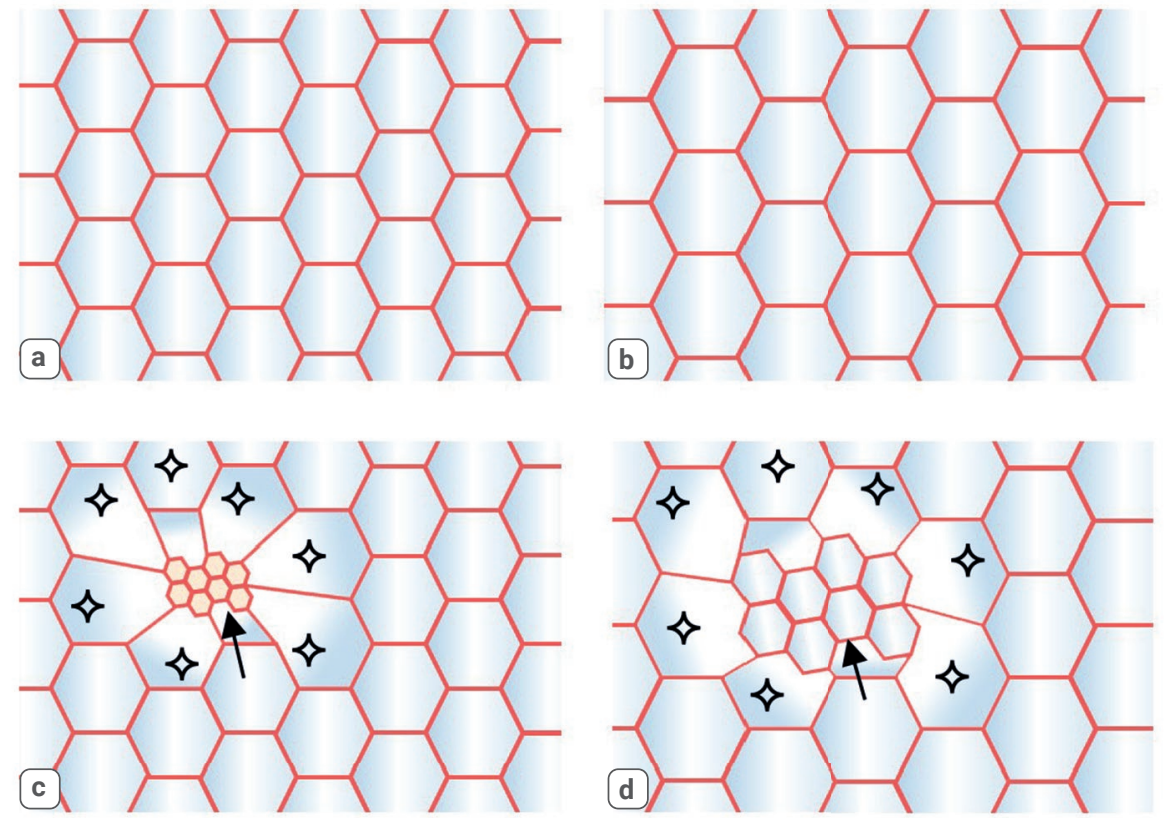

4. Représentation schématique de l'expansion des alvéoles dans un poumon normal ou lésé. Les alvéoles pulmonaires normales (a), schématisées par des structures hexagonales, sont aérées et subissent une expansion relativement homogène en inspiration (b). Dans un poumon lésé (c), des zones hétérogènes du poumon présentent des alvéoles vidées d'air (flèche). À cause de leur instabilité mécanique, ces alvéoles se rouvrent à l'inspiration (d) et se referment à chaque cycle respiratoire, ce qui soumet leurs parois à de fortes contraintes de cisaillement. Les alvéoles adjacentes $(\diamond)$ subissent également un stress mécanique élevé en expiration, avec des contraintes dynamiques à chaque respiration.

\section{Observer les poumons grâce à l'imagerie par contraste de phase}

L'un des symptômes d'une infection par le SARS-CoV-2 est le développement d'une détresse respiratoire aigüe. Cette détresse est caractérisée par une inflammation des poumons entrainant un trouble des échanges gazeux et une rigidité plus grande des tissus pulmonaires, rendant la respiration plus difficile. Cette détresse respiratoire requiert souvent une ventilation mécanique du patient par un respirateur artificiel.
Mais dans certains cas, cette ventilation artificielle du patient peut entrainer ou aggraver des lésions pulmonaires. Le mécanisme microscopique amenant à ces lésions est mal compris, même s'il est généralement admis qu'elles sont causées par le stress supplémentaire résultant de la contrainte mécanique imposée au tissu pulmonaire par la respiration. L'épaisseur des parois des alvéoles pulmonaires étant voisine de $15 \mu \mathrm{m}$ (micromètres), l'étude de ces mécanismes microscopiques requiert une résolution meilleure que $10 \mu \mathrm{m}$ et n'est pas réalisable avec les techniques

\section{Imagerie par contraste de phase grâce au rayonnement synchrotron}

La phase d'un faisceau de rayons $X$ évolue différemment selon que le faisceau se propage dans le vide ou dans de la matière. L'imagerie par contraste de phase utilise cette propriété pour observer des changements de densité ou de composition trop faibles pour provoquer une absorption importante, mais suffisante pour engendrer un déphasage des rayons $X$. Cette technique requiert que les rayons $X$ utilisés soient produits de manière cohérente, ce qui est possible sur les sources de rayonnement synchrotron [8]. 

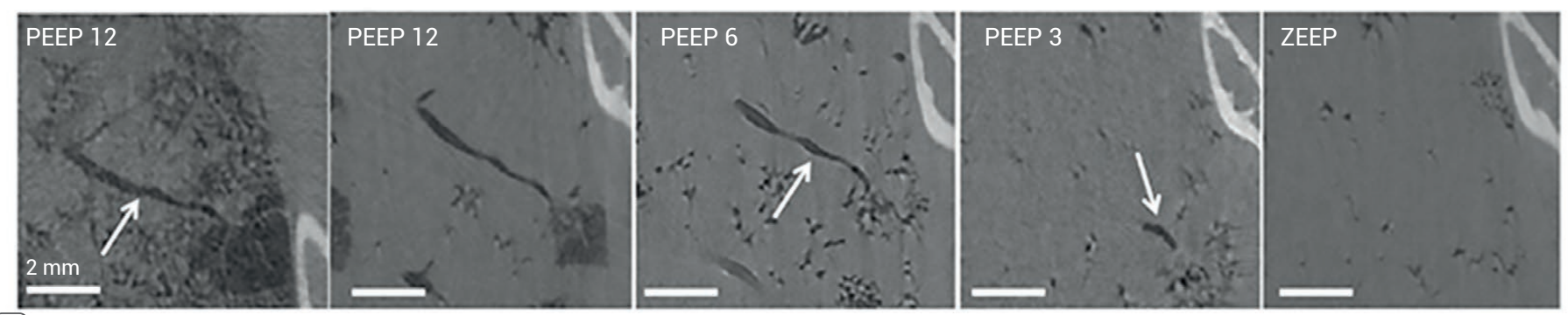

a

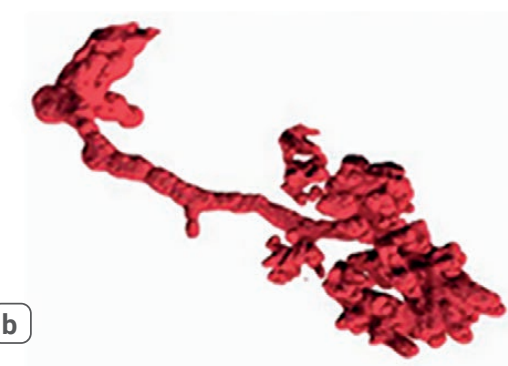

PEEP 12

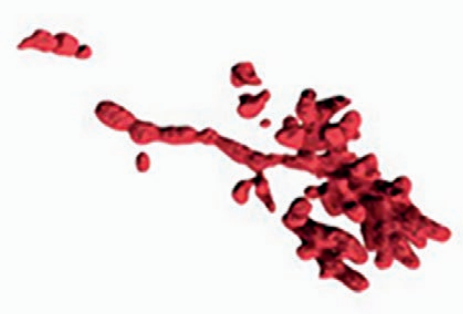

PEEP 6

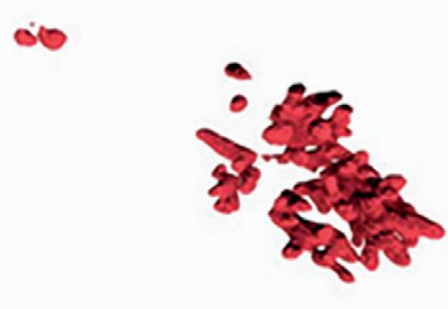

PEEP 3

5. Fermeture de petites bronches dans un poumon lésé.

(a) Images prises par imagerie à contraste de phase montrant une tranche obtenue par tomographie. (b) Représentation 3D de bronchioles se fermant lorsque la pression est réduite, dans un modèle in vivo de détresse respiratoire. ZEEP signifie "zero end-expiratory pressure", et PEEP signifie "positive end-expiratory pressure". Figure adaptée de [10].

$>>$

d'imagerie X conventionnelles. Par ailleurs, le tissu pulmonaire absorbe peu les rayons $\mathrm{X}$, ne générant qu'un faible contraste dans les images tomographiques classiques obtenues par atténuation du faisceau incident. En revanche, les nombreuses interfaces entre le tissu et l'air diffractent beaucoup les rayons X. L'imagerie par contraste de phase (encadré 3, p. 7) est par conséquent une solution adaptée à l'imagerie microscopique pulmonaire.

L'imagerie par contraste de phase a été utilisée pour étudier chez des lapins anesthésiés les ouvertures et fermetures d'alvéoles pulmonaires au cours du cycle respiratoire, un mécanisme potentiel de lésion de la ventilation mécanique. Alors que dans un poumon normal les alvéoles restent aérées au cours de tout le cycle respiratoire (fig. $4 \mathrm{a}$ et b), la fermeture et la réouverture des alvéoles dans un poumon lésé sous ventilation mécanique produisent des forces d'étirement anormalement élevées sur les structures tissulaires, à l'origine de lésions mécaniques et d'une stimulation de l'inflammation. Ces études en imagerie de contraste de phase ont montré que des alvéoles adjacentes et soumises à la même pression peuvent avoir un comportement dynamique très différent au cours du cycle respiratoire, certaines se rouvrant alors qu'au même moment d'autres se ferment (fig. 4c et d). Autrement dit, l'ouverture et la fermeture successive des zones alvéolaires au cours du cycle respiratoire ne sont pas seulement déterminées par la pression à laquelle les alvéoles sont soumises, mais se produisent avec une constante de temps caractéristique. Ceci implique qu'en manipulant la durée pendant laquelle le patient expire, il est possible de réduire les phénomènes d'ouverture et de fermeture alvéolaires. Ces résultats ont des implications importantes pour améliorer les protocoles de ventilation artificielle chez les patients atteints de détresse respiratoire, afin de les rendre moins invasives [9].

En poussant l'étude plus loin [10], il a été démontré que les petites bronches ont tendance à se fermer dans un poumon lésé (fig. 5). Des études théoriques avaient prédit ce phénomène par des instabilités dans la fine couche de liquide qui tapisse les parois des bronchioles. Lorsque ce fluide s'épaissit, par exemple en présence d'un œdème dans un poumon atteint par la détresse respiratoire aigüe, les forces de tension superficielle résultantes peuvent entrainer une occlusion de la bronchiole sur une certaine distance. La fermeture des petites bronches dans le poumon lésé avait longtemps été suspectée, mais jamais observée in vivo. Il en résulte un piégeage
L'imagerie fonctionnelle pulmonaire avec une source de rayons $\mathrm{X}$ synchrotron permet de visualiser le cheminement de l'air inspiré dans les poumons, et donc de mieux comprendre l'impact fonctionnel de la ventilation mécanique sur le poumon. 
de l'air dans les alvéoles situées en aval, menant à leur affaissement progressif. Ce phénomène a été observé et caractérisé directement à l'échelle microscopique par imagerie de contraste de phase dans un modèle expérimental de détresse respiratoire [10]. L'implication de ces études porte sur les stratégies de ventilation mécanique chez le patient : minimiser le temps pendant lequel la pression respiratoire est abaissée au cours de l'expiration peut prévenir la fermeture des petites voies aériennes. Grâce à cette technique d'imagerie, il a aussi été possible dans une autre étude de visualiser la progression de lésions induites par la ventilation artificielle dans les poumons de souris [11].

Cette technique d'imagerie est aussi utilisée pour mieux comprendre pourquoi certains patients atteints de Covid-19 ont un très faible niveau d'oxygène dans le sang. Ces patients souffrent d'anomalies de flux sanguin pulmonaire et d'un mauvais rapport entre la ventilation et le flux sanguin, empêchant les échanges gazeux et provoquant une hypoxémie ${ }^{(\mathrm{d})}$. Pour comprendre les mécanismes de ces anomalies, qui peuvent notamment être dues à la formation de microcaillots dans la circulation pulmonaire ou à une vascularite ${ }^{(e)}$, l'imagerie de contraste de phase post mortem (c'est-à-dire chez des patients décédés de la Covid-19) permet de décrire la microstructure et l'architecture vasculaire dans le poumon, en même temps que celle des voies aériennes, à une résolution meilleure que $1 \mu \mathrm{m}$, aidant ainsi à mieux comprendre les mécanismes de la maladie.

Par ailleurs, une autre technique d'imagerie synchrotron donne accès à la dynamique des variations de volume sanguin et de la ventilation pulmonaire régionale au cours du cycle respiratoire dans des modèles expérimentaux de ventilation mécanique chez l'animal [12]. Ici, le choix d'une valeur spécifique de l'énergie des rayons $\mathrm{X}$ incidents permet de quantifier un traceur gazeux, le xénon, dans l'air inspiré et en même temps un produit de contraste iodé dans le sang. Ainsi, l'imagerie fonctionnelle pulmonaire avec une source de rayons $\mathrm{X}$ synchrotron permet de visualiser le cheminement de l'air inspiré dans les poumons et donc de mieux comprendre l'impact fonctionnel de la ventilation mécanique sur le poumon.

\section{Références}

$1 \cdot$ M.W. Bowler et D. Nurizzo, « Le rayonnement synchrotron : comprendre la relation structurefonction des macromolécules biologiques », Reflets de la physique 34-35 (2013) 48-53.

2. Jun Lan et al., "Structure of the SARS-CoV-2 spike receptor-binding domain bound to the ACE2 receptor", Nature 581 (2020) 215-220.

$3 \cdot \mathrm{L}$. Zhang et al., "Crystal structure of SARS-CoV-2 main protease provides a basis for design of improved $\alpha$-ketoamide inhibitors", Science 368 (2020) 409-412.

4. www.wwpdb.org/pdb?id=pdb_00006lu7

5 Zhenming Jin et al., "Structure of Mpro from SARS-CoV-2 and discovery of its inhibitors", Nature 582 (2020) 289-293.

6• Voir par exemple : https://cutt.ly/synchrotron-soleil-fr-recherchesurlecovid19, et https://cutt.ly/esrf-eu-covid-19-scientific-research .

7. "Reflection of the ILL director, Pr Helmut Schober, in the context of the pandemic", dans www.ill.eu/news-press-events/news/general-news/coronavirus-covid-10 .

8. J. Baruchel et al., Reflets de la physique 34-35 (2013) 32-37.

9. L. Broche et al., "Dynamic Mechanical Interactions Between Neighboring Airspaces Determine Cyclic Opening and Closure in Injured Lung", Critical Care Medicine 45 (2017) 687-694.

10 - L. Broche et al., "Individual airway closure characterized in vivo by phase-contrast CT imaging in injured rabbit lung", Critical Care Medicine 47 (2019) e774-e781.

$11 \cdot$ G. Lovric et al., "Spatial Distribution of Ventilator Induced Lung Injury at the $\mu \mathrm{m}$-Scale. An In-Vivo Synchrotron Phase-Contrast Microscopy Study in BALB/c Mice". AJRCRM, 197 (2018) A7526.

12 L. Porra et al., "Synchrotron imaging shows effect of ventilator settings on intrabreath cyclic changes in pulmonary blood volume", Am. J. Respir. Cell Mol. Biol. 57 (2017) 459-467.

\section{Conclusion}

Les travaux de diffraction et d'imagerie effectués sur accélérateurs depuis le début de la crise sanitaire ont donc contribué à la lutte contre les effets du SARS-CoV-2. La cristallographie a permis de déterminer la structure de certaines des protéines produites par ce virus et de chercher des molécules capables de l'inhiber. L'imagerie par contraste de phase a apporté des informations utiles sur la manière dont les poumons se comportent lorsqu'un patient est mis sous respiration artificielle. Elle a aussi permis de mieux comprendre comment la Covid-19 affecte les poumons, afin de mieux lutter contre elle. Ces recherches apportent aux soignants des outils pour les aider dans leurs efforts contre cette maladie.

(a) Un « virion » est un virus non lié à une cellule hôte.

(b) ACE2 : enzyme de conversion de l'angiotensine, un récepteur situé à la surface de nombreuses sortes de cellules, qui est en particulier impliqué dans la régulation de la pression artérielle. L'ACE2 est le point d'entrée de certains coronavirus dans les cellules.

(c) Une "protéase " (aussi appelée "peptidase ") est une enzyme capable de briser certaines protéines et en particulier les peptides.

(d) L'hypoxémie est la diminution de la pression partielle de l'oxygène transporté dans le sang.

(e) Vascularite : atteinte inflammatoire des vaisseaux.

Pour en savoir plus

- Numéro spécial n³4-35 de

Reflets de la physique, "La lumière synchrotron au service de la science et de la société », paru en juin 2013.

- https://cerncourier.com/a/synchrotronson-the-coronavirus-frontline/

- https://cerncourier.com/a/neutronsources-join-the-fight-against-covid-19/.

- «Quel rôle pour la cristallographie dans la lutte contre le Covid-19? » (site de l'Association française de cristallographie), https://cutt.ly/afc-role-cristallographielutte-covid.

- Site du Synchrotron SOLEIL :

https://www.synchrotron-soleil.fr/

- Site de la Source européenne de rayonnement synchrotron (ESRF) : www.esrf.eu/ 